\title{
Molecular dynamics simulation of the interaction of ethanol-water mixture with a Pt surface
}

\author{
Kholmirzo Kholmurodov ${ }^{1,2^{*}}$, Ermuhammad Dushanov ${ }^{1,3}$, Kenji Yasuoka $^{4}$, Hagar Khalil ${ }^{5}$, \\ Ahmed Galal $^{5}$, Sameh Ahmed ${ }^{6}$, Nasser Sweilam ${ }^{6}$, Hatem Moharram ${ }^{6}$ \\ ${ }^{1}$ Laboratory of Radiation Biology, Joint Institute for Nuclear Research, Dubna, Russia; *Corresponding Author: $\underline{\text { mirzo@jinr.ru }}$ \\ ${ }^{2}$ Dubna International University, Dubna, Moscow Region, Russia; \\ ${ }^{3}$ Institute of Nuclear Physics, Tashkent, Uzbekistan; \\ ${ }^{4}$ Department of Mechanical Engineering, Keio University, Yokohama, Japan; \\ ${ }^{5}$ Chemistry Department, Faculty of Science, Cairo University, Cairo, Egypt; \\ ${ }^{6}$ Mathematics Department, Faculty of Science, Cairo University, Cairo, Egypt.
}

Received 9 November 2011; revised 29 November 2011; accepted 14 December 2011.

\begin{abstract}
An analysis of the molecular dynamics of ethanol solvated by water molecules in the absence and presence of a Pt surface has been performed using DL_POLY_2.19 code. The structure and diffusion properties of an ethanol-water system have been studied at various temperatures from 250 to $600 \mathrm{~K}$. We have measured the self-diffusion coefficients of the $50: 50 \%$ ethanol-water solution; in the absence of a Pt surface our results show an excellent agreement-within an error of $7.4 \%$-with the experimental data. An increase in the self-diffusion coefficients with the inclusion of a Pt surface has been observed. The estimation of the diffusion coefficients of both water and ethanol in the presence of a $\mathrm{Pt}$ surface shows that they obey the Arrhenius equation; the calculated activation energies of diffusion of ethanol and water are 2.47 and 2.98 $\mathrm{Kcal} / \mathrm{mole}$, respectively. The radial distribution function graphs and density profiles have been built; their correlations with the self-diffusion coefficients of both ethanol and water molecules are also illustrated.
\end{abstract}

Keywords: Molecular Dynamics Simulations; Ethanol Molecule; Water Active Solvent; Diffusion Coefficient; Pt Surface; RDF Graphs

\section{INTRODUCTION}

In recent years, there has been a great interest in studying the chemical and physical properties of ethanol on a Pt surface, as ethanol is one of the most important renewable fuels [1]. The intensive utilization of fossil fuels has led to an increase in the generation of polluting gases released into the atmosphere, which has caused changes in the global climate. The solution to this problematic depends on how the development and implementation of technologies based on alternative sources of energy will be undertaken. Among the renewable energetic resources, ethanol (ethyl alcohol, bioethanol) is the most practical liquid biofuel - both as a fuel and a gasoline enhancer. It is not toxic, does not contaminate water sources [2], and can be produced in large quantities from agricultural products or biomass, which will not change the natural balance of carbon dioxide in the atmosphere in contrast to the use of fossil fuels [3].

Ethanol has been considered in recent years, and it has a lot of applications. The most popular application is fuels because of a decrease in the available petroleum resources. For ethanol to be a fuel, the water content in ethanol should be less than $1.3 \%$ [4], which is hard to reach by crystallization. Pervaporation separation is a valuable method that can save money, and therefore much research is focused on it. Direct ethanol fuel cells (DEFCs) are another important application for the conversation of chemical energy to electricity $[5,6]$. Water and ethanol can be used not only in fuels, but also in other applications, such as being a solvent to accelerate the aging of some polymeric materials [7] and being used in commercial cooling systems because of their good thermophysical and technological characteristics [8]. An alcohol-water mixture often shows quite different properties than the corresponding pure components. Of particular interest are the structure and diffusion properties, which play important roles in the theoretical study and technological applications involving mass transfer [9]. In addition, from a microscopic viewpoint, the knowledge of solution structure behavior is very 
fundamental to understanding and elucidating the mixture diffusion phenomenon.

Molecular dynamics simulation is a powerful tool in investigating the structure properties of solutions at the molecular level, and it has been widely used to study aqueous solutions [10,11]. Metal surfaces are often used in the synthesis of oxygen containing compounds, such as alcohols, and in the degradation of these oxygencontaining compounds, where carbon-carbon (C-C) and carbon-oxygen $(\mathrm{C}-\mathrm{O})$ bond formation and breakage are the elementary steps in this type of process, and the metal surface plays a primary role in the efficiency and selectivity of these steps [12]. Platinum is the most known catalyst for the oxidation of such molecule. It is known to activate the dissociative adsorption of ethanol at an appreciable rate [13]. Thus, studying the adsorption of ethanol on a platinum surface can give more information about the kinetics of this process. Molecular dynamics (MD) simulation is one of the most important computational tools to study the liquid-surface interaction. At a high temporal resolution, MD processes may provide information about the dynamics of the system and the events which take place on the surface within a few picoseconds [14]. The application of molecular dynamics to liquids or solvent-solute systems allows the computation of properties such as diffusion coefficients or radial distribution functions for use in statistical mechanical treatments [15].

Few researches were performed on ethanol-water surface. C. Zhang and X. Yang studied the structure behavior and diffusion properties for an ethanol-water solution and investigated the concentration dependence of properties [9]. Wang Yao-Chun et al. used MD simulation to investigate the behavior of pure water molecules, ethanol molecules, and water-ethanol mixture with various weight fractions inside Au nanotubes [7]. D. J. Cooke et al. studied the interface between the $\{10 \overline{1} 4\}$ surface of calcite and pure ethanol, pure water, and 50:50 mixture (by amount) of water and ethanol [16].

To the best of our knowledge, little is known from literature surveys about the interesting ethanol-water interactions in the presence of Pt surfaces. In the present work, using the MD method, we have simulated ethanolwater system in the absence and presence of a platinum surface through a wide temperature range-from 250 to $600 \mathrm{~K}$ - and calculated self-diffusion coefficients. The enhancement of the self-diffusion coefficients of both water and ethanol molecules correlating with the ethanol-water structure has been well established in the presence of a Pt surface.

\section{SIMULATION METHOD}

We have studied the molecular dynamics of a wa- ter-ethanol solution system in the absence and presence of a platinum surface using the DL_POLY_2.19 code, which was developed by the Molecular Simulation Group at the Daresbury Laboratory (England) with the support of the Research Council for the Engineering and Physical Sciences (project CCP5 of the simulation of condensed phases). DL POLY is a general-purpose MD simulation package developed by W. Smith, T. P. Forester and I. T. Todorov $[17,18]$.

\subsection{Simulation Details}

Ethanol and water molecules are described using the force field from the DL POLY data base $[17,18]$, where bonding, angular, and dihedral parameters are incorporated into standard molecular mechanics potentials. Nonbonding interactions are accounted for via Lennard-Jones (LJ) potentials and Coulombic interactions based on the partial charges associated in each atom. For water, the SPC model is used. The computer simulations have been performed for a MD cell of a volume $V=$ $(54.92,54.92,63.8) \AA^{3}$ under the energy and temperature control at $T=298 \mathrm{~K}$ and other temperatures. Starting with a 50:50 (by molecules) water-ethanol solution, with a corresponding density of about $0.78 \mathrm{~g} / \mathrm{cm}^{3}$ and $588 \mathrm{Pt}_{4}$ molecules of 2352 atoms, the total number of atoms in the system was $N=16176$; the chemical bonds are constrained within a flexible bond with a length of about $1 \AA$. The integration of the equations of motion was performed using the Verlet integration scheme in quaternion. The integration step was $1 \mathrm{fs}$ (fem- tosecond); a microcanonical (nvt) ensemble was used for the simulated system, and the Nose-Hoover algorithm was employed to keep the desired temperature. The intermolecular chemical bonds were estimated on the basis of the Shake algorithm with an accuracy of $10^{-8}$. The Ewald summation with a convergence parameter of $10^{-6}$ was used for the calculation of electrostatics forces in the periodic system. The total number of steps was 100,000 for each temperature, and all simulations were periodic in three dimensions.

The configuration energy of the molecular model is represented as a sum of the energies of the bonding $\left(E_{v a l}\right)$ and non-bonding $\left(E_{n b}\right)$ interactions:

$$
E=E_{v a l}+E_{n b} .
$$

The energy of the valence (bonding) interactions $E_{v a l}$ is given by the following formula:

$$
E_{\text {val }}=E_{\text {bond }}+E_{\text {ang }}+E_{\text {dih }}+E_{\text {teth }},
$$

where $E_{\text {bond }}$ is the energy of chemical bonds, $E_{\text {ang }}$ is the energy of angular bonds, $E_{d i h}$ is the energy of dihedral bonds, and $E_{\text {teth }}$ is tether energy.

The energy of the non-valence (non-bonded) interac- 
tions is a sum of the energies of the van-der-Waals $(v d W)$, electrostatics (Coulomb), and hydrogen bonds:

$$
E_{n b}=E_{v d W}+E_{\text {coul }} \text {. }
$$

During the MD simulations, the following potential types, which represent the topology of the molecular field for an ethanol-water system, were used [18]:

Harmonic bond potential:

$$
U\left(r_{i j}\right)=\frac{1}{2} K\left(r_{i j}-r_{0}\right)^{2} .
$$

Harmonic bond angle potential:

$$
U\left(\theta_{i j k}\right)=\frac{1}{2} K\left(\theta_{i j k}-\theta_{0}\right)^{2} .
$$

Harmonic dihedral potential:

$$
U\left(\varphi_{i j k n}\right)=\frac{1}{2} K\left(\varphi_{i j k n}-\varphi_{0}\right)^{2}
$$

The Lennard-Jones potential:

$$
U_{i j}\left(r_{i j}\right)=4 \varepsilon_{i j}\left[\left(\frac{\sigma_{i j}}{r_{i j}}\right)^{12}-\left(\frac{\sigma_{i j}}{r_{i j}}\right)^{6}\right] .
$$

Coulombic interaction:

$$
U\left(r_{i j}\right)=\frac{1}{4 \pi \varepsilon_{0}} \frac{q_{i} q_{j}}{r_{i j}} .
$$

Quadratic tether potential:

$$
U(r)=\frac{1}{2} k r^{2}+\frac{1}{4} k^{\prime} r^{4} .
$$

The tether potential suggests that the momentum wills no longer be a conserved quantity of the simulation. The force on the atom " $i$ " arising from the tether potential is obtained using the general formula:

$$
\underline{F}_{i}=-\frac{1}{r_{i 0}}\left[\frac{\partial}{\partial r_{i 0}} U_{i 0}\right] \underline{r}_{i 0},
$$

where $\sigma_{i j}$ is the size parameter, $\varepsilon_{i j}$ the energy parameter, $\sigma_{i j}=\left(\sigma_{i}+\sigma_{j}\right) / 2$ and $\varepsilon_{i j}=\sqrt{\varepsilon_{i} \varepsilon_{j}}, q_{i}$ is the charge of site $i$ and $r_{i j}$ the distance between sites $i$ and $j$. We choose the values of $k=0.2$ and $k^{\prime}=0.4$ to avoid the destruction of our surface during heating process.

Water was represented by the constrained OW-HW bond potential; thus a SPC model was used. Tables 1-4 contain charges, bond lengths and intermolecular Lennard-Jones parameters for ethanol, water molecules, and a Pt surface respectively.

\subsection{Metal Potential}

DL POLY 2 includes density-dependent potentials suitable for calculating the properties of metals. One of the potentials used in our MD simulation is the one described by Sutton and Chen (SC or st-ch) [19]:

$$
\begin{gathered}
U=\sum_{i} U_{i}, \\
U_{i}=\left[\frac{1}{2} \sum_{j \neq i}\left(\frac{a}{r_{i j}}\right)^{n}-c \sqrt{\rho_{i}}\right] .
\end{gathered}
$$

Here $\rho_{i}$ is a density-like term for atom $i$ :

$$
\rho_{i}=\sum_{j \neq i}\left(\frac{a}{r_{i j}}\right)^{m} .
$$

Here the potential has three dimensionless parameters adjustable for the material. They are $c, n$, and $m$, and can be chosen for various materials, especially metals. The variable $\varepsilon$ sets the energy scale; $a$ is the lattice constant. Table 3 contains the potential parameters of $\mathrm{SC}$ of $\mathrm{Pt}$ surface which were used.

\subsection{Simulation System}

We have two simulated systems one for 50:50\% ethanol-water in the absence and the other in presence of platinum surface. The number of molecules of Pt surface was kept constant (588 molecules), the density of waterethanol mixture in the two systems was $0.78 \mathrm{~g} / \mathrm{cm}^{3}$. The temperature of the system was varied from 250 to $600 \mathrm{~K}$ using the annealing process starting with $250 \mathrm{~K}$ then

Table 1. The effective charges of atoms of ethanol, water and a Pt surface.

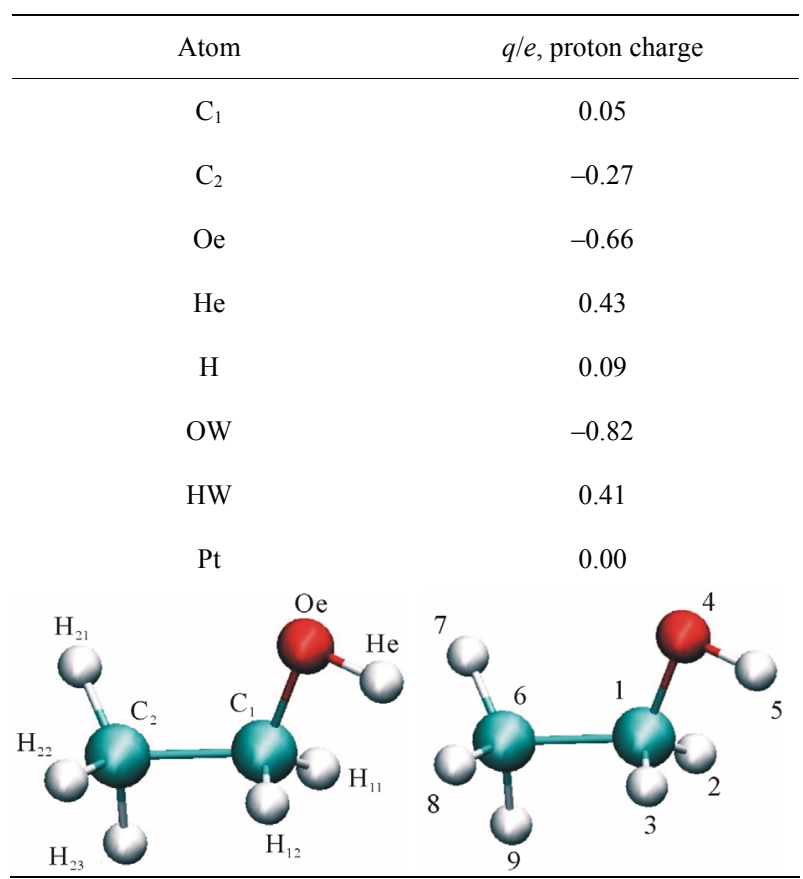


Table 2. The potential parameters used for ethanol molecules.

\begin{tabular}{|c|c|c|}
\hline \multirow{2}{*}{ Bond } & \multicolumn{2}{|c|}{ Harmonic bond potential $=K\left(r_{i j}-r_{0}\right)^{2} / 2$} \\
\hline & $K\left(\mathrm{kcal} \cdot \mathrm{mol}^{-1} \cdot \AA\right)$ & $r_{0}(\AA)$ \\
\hline $\mathrm{C}_{1}-\mathrm{C}_{2}$ & 222 & 1.52 \\
\hline $\mathrm{C}-\mathrm{H}$ & 309 & 1.11 \\
\hline $\mathrm{C}_{1}-\mathrm{Oe}$ & 428 & 1.42 \\
\hline $\mathrm{Oe}-\mathrm{He}$ & 545 & 0.94 \\
\hline \multirow{2}{*}{ Group } & \multicolumn{2}{|c|}{ Angular potential $=K\left(\theta_{i j k}-\theta_{0}\right)^{2} / 2$} \\
\hline & $K\left(\mathrm{kcal} \cdot \mathrm{mol}^{-1} \cdot \mathrm{rad}^{-2}\right)$ & $\theta_{0}\left({ }^{\circ}\right)$ \\
\hline $\mathrm{H}_{1}-\mathrm{C}_{1}-\mathrm{Oe}$ & 45.90 & 109.44 \\
\hline $\mathrm{H}_{1}-\mathrm{C}_{1}-\mathrm{C}_{2}$ & 34.60 & 109.46 \\
\hline $\mathrm{H}_{1}-\mathrm{C}_{1}-\mathrm{H}_{1}$ & 35.50 & 120.00 \\
\hline Oe- $\mathrm{C}_{1}-\mathrm{C}_{2}$ & 75.70 & 109.00 \\
\hline $\mathrm{He}-\mathrm{Oe}-\mathrm{C}_{1}$ & 57.50 & 109.50 \\
\hline $\mathrm{H}_{2}-\mathrm{C}_{2}-\mathrm{H}_{2}$ & 35.50 & 109.50 \\
\hline $\mathrm{H}_{2}-\mathrm{C}_{2}-\mathrm{C}_{1}$ & 34.60 & 109.46 \\
\hline \multirow{2}{*}{ Group } & \multicolumn{2}{|c|}{ Dihedral potential $=K\left(\varphi_{i j k n}-\varphi_{0}\right)^{2} / 2$} \\
\hline & $K\left(\mathrm{kcal} \cdot \mathrm{mol}^{-1}\right)$ & $\varphi_{0}\left({ }^{\circ}\right)$ \\
\hline $\mathrm{C}_{2}-\mathrm{C}_{1}-\mathrm{Oe}-\mathrm{He}$ & 1.30 & 180 \\
\hline $\mathrm{H}_{12}-\mathrm{C}_{1}-\mathrm{Oe}-\mathrm{He}$ & 0.14 & 60 \\
\hline $\mathrm{H}_{11}-\mathrm{C}_{1}-\mathrm{Oe}-\mathrm{He}$ & 0.14 & -60 \\
\hline $\mathrm{Oe}-\mathrm{C}_{1}-\mathrm{C}_{2}-\mathrm{H}_{21}$ & 0.16 & 180 \\
\hline Oe- $\mathrm{C}_{1}-\mathrm{C}_{2}-\mathrm{H}_{22}$ & 0.16 & 60 \\
\hline $\mathrm{Oe}-\mathrm{C}_{1}-\mathrm{C}_{2}-\mathrm{H}_{23}$ & 0.16 & -60 \\
\hline $\mathrm{H}_{11}-\mathrm{C}_{1}-\mathrm{C}_{2}-\mathrm{H}_{21}$ & 0.16 & -60 \\
\hline $\mathrm{H}_{11}-\mathrm{C}_{1}-\mathrm{C}_{2}-\mathrm{H}_{22}$ & 0.16 & 180 \\
\hline $\mathrm{H}_{11}-\mathrm{C}_{1}-\mathrm{C}_{2}-\mathrm{H}_{23}$ & 0.16 & 60 \\
\hline $\mathrm{H}_{12}-\mathrm{C}_{1}-\mathrm{C}_{2}-\mathrm{H}_{21}$ & 0.16 & 60 \\
\hline $\mathrm{H}_{12}-\mathrm{C}_{1}-\mathrm{C}_{2}-\mathrm{H}_{22}$ & 0.16 & -60 \\
\hline $\mathrm{H}_{13}-\mathrm{C}_{1}-\mathrm{C}_{2}-\mathrm{H}_{23}$ & 0.16 & -180 \\
\hline
\end{tabular}

Table 3. The Sutton-Chen (st-ch) potential parameters of platinum $(\mathrm{Pt})$.

\begin{tabular}{ccccc}
\hline$\varepsilon\left(\mathrm{kcal} \cdot \mathrm{mol}^{-1}\right)$ & $a(\AA)$ & $N$ & $m$ & $C$ \\
\hline 0.226 & 3.92 & 11.0 & 7.0 & 71.336 \\
\hline
\end{tabular}

Table 4. Intermolecular Lennard-Jones parameters for ethanol, water, and a $\mathrm{Pt}$ surface, water, and $588 \mathrm{Pt}_{4}$ molecules containing a total of 16176 atoms in a volume $V=(54.92,54.92$, 63.801) $\AA^{3}$.

\begin{tabular}{ccc}
\hline Group & $\varepsilon\left(\mathrm{kcal} \cdot \mathrm{mol}^{-1}\right)$ & $\sigma(\AA)$ \\
\hline C-C & 0.12 & 3.30 \\
C-H & 0.00 & 2.54 \\
C - Oe & 0.16 & 3.08 \\
H-H & 0.00 & 1.78 \\
H-O & 0.00 & 2.32 \\
Oe-Oe & 0.20 & 2.85 \\
C-Pt & 0.94 & 2.90 \\
Oe-Pt & 0.92 & 2.70 \\
C-OW & 0.14 & 3.43 \\
Oe-OW & 0.18 & 3.20 \\
OW-OW & 0.22 & 3.17 \\
\hline
\end{tabular}

raises the temperature by $25 \mathrm{~K}$ in each simulation.

\subsection{Metal Surface}

The metallic substrate used was cubic Pt, which has the formula $\mathrm{Pt}_{4}$; and it was arranged in 6 layers numbering a total of 2352 atoms. The surface area was $60.38 \AA^{2}$; and the lattice constant was $a=3.923 \AA$. All the parameters of platinum were taken from EIM databases and datasets website supported by the Russian Foundation for Basic Research [20].

\section{RESULTS AND DISCUSSION}

Figure 1 shows a snapshot of an equilibrated state of a water-ethanol-Pt system with a total of $N=2892$ molecules where the locations of water and ethanol molecules on the surface are clearly seen.

From the MD simulation results, we first estimated the self-diffusion coefficient $D$ for both ethanol and water molecules. We measured $D$ in the absence and presence of a $\mathrm{Pt}$ surface. The diffusion coefficient $D$ was estimated for a 50:50\% ethanol-water solution at $298 \mathrm{~K}$ in the absence of $\mathrm{Pt}$; the results were compared with those of experimental [9] and other MD simulations [21, 22]. The diffusion coefficient of ethanol molecules $D_{e}$ derived from [21] was ranged from 0.98 to $1.0 \times 10^{-9}$ $\mathrm{m}^{2} \cdot \mathrm{s}^{-1}$. The experimental $D_{e}$, at the same conditions as in our simulations, was about $0.7 \times 10^{-9} \mathrm{~m}^{2} \cdot \mathrm{s}^{-1}$; from our simulation, we have obtained $D_{e}$ to be around $0.65 \times$ $10^{-9} \mathrm{~m}^{2} \cdot \mathrm{s}^{-1}$, which is in agreement with experimental 


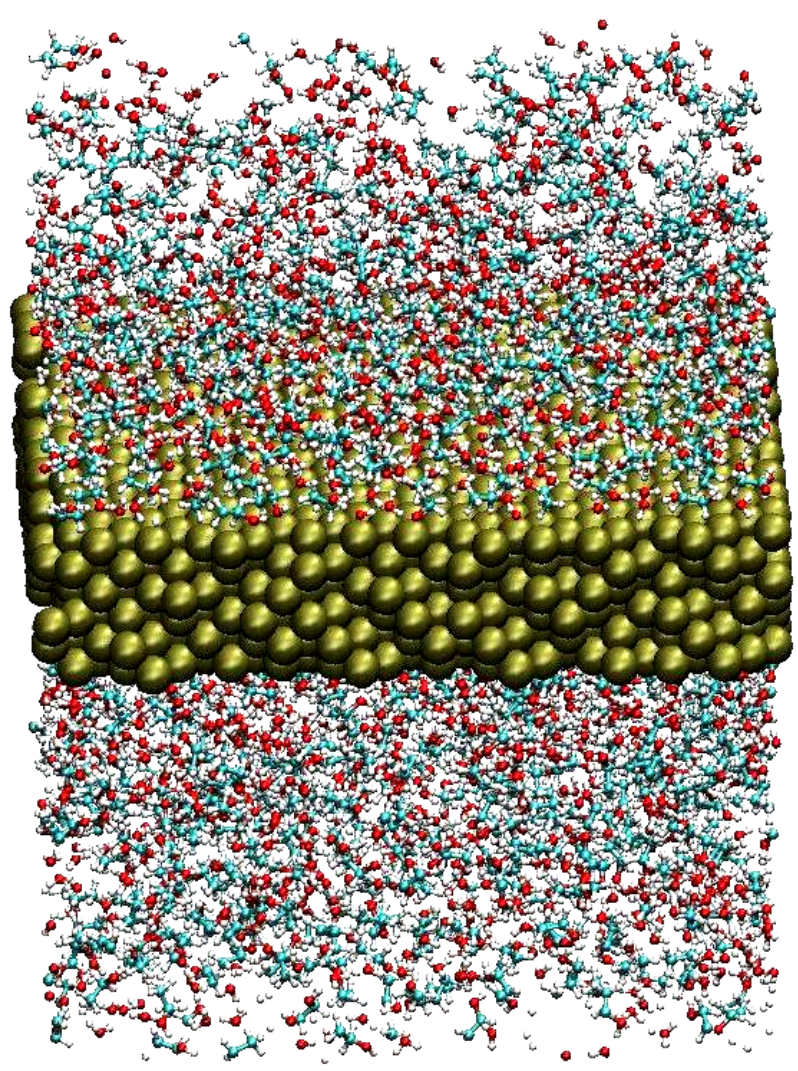

Figure 1. Snapshot of an ethanol-water-Pt system at $298 \mathrm{~K}$ which consists of 1152 ethanol, 1152 water, and $588 \mathrm{Pt}_{4}$ molecules containing a total of 16176 atoms in a volume $V=$ $(54.92,54.92,63.80) \AA^{3}$.

results with an accuracy of $7.4 \%$. At the same time, the self-diffusion coefficient of water $D_{w}$ is about $1.5 \times 10^{-9}$ $\mathrm{m}^{2} \cdot \mathrm{s}^{-1}$, which is in good agreement with the results of [22], but still higher than the experimental one: $0.88 \times$ $10^{-9} \mathrm{~m}^{2} \cdot \mathrm{s}^{-1}$.

As regards the presence of a Pt surface, neither simulation nor experimental data are available in the literature. From our MD simulations, the diffusion coefficients $D_{e}$ and $D_{w}$ of both ethanol and water have to increase in comparison with the results on the absence of a Pt surface. $D_{e}$ reaches $1.07 \times 10^{-9} \mathrm{~m}^{2} \cdot \mathrm{s}^{-1}$ and $D_{w}$ reaches $2.1 \times 10^{-9} \mathrm{~m}^{2} \cdot \mathrm{s}^{-1}$, which indicates that the presence of a Pt surface essentially affects the mobility of the ethanol and water molecules. The ethanol and water molecules form an adsorption layer on the Pt surface. So, we observe competition between the adsorption and diffusion processes as the molecules reach the metal surface; the surface and bulk molecules exhibit different behavior.

Next, in Figure 2 we present the self-diffusion coefficients for water (a) and ethanol (b). The temperature of the system was varied, and the temperature effect on the self-diffusion coefficient for both water and ethanol molecules were investigated. It is shown that the diffu-

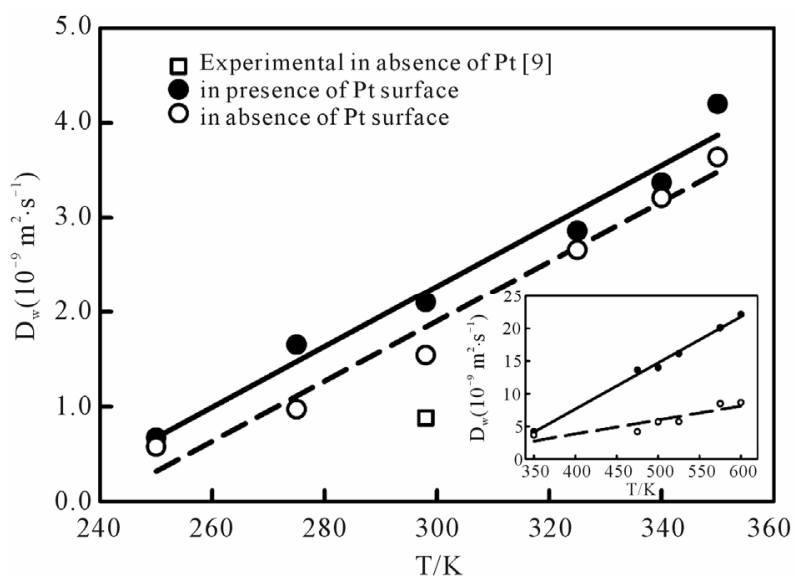

(a)

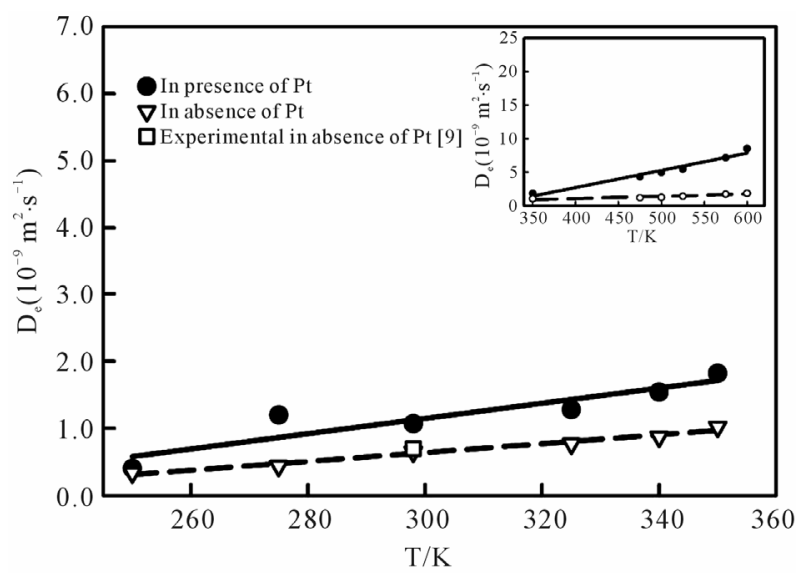

(b)

Figure 2. A temperature dependence of the diffusion coefficient for water (a) and ethanol (b). The solid line represents an ethanol-water-Pt system in the presence of a Pt surface; the dashed lines represent an ethanol-water system in the absence of a Pt surface.

sion coefficients decrease with decreasing temperature, which is consistent with the formation of longer $\mathrm{H}$-bonded chains at low temperatures. Initially, at a low temperature $(250 \mathrm{~K})$, the presence of a Pt surface had no effect on the value of $D$ for both water and ethanol. After that, the role of Pt appears, and the enhancement of the $D$ value in the presence of a Pt surface is clearly observed - especially at higher temperatures, which is consistent with Pt being a good catalyst for such molecules.

It is believed that the self-diffusion coefficient follows an Arrhenius-like relation with temperature [23]. The relationship is

$$
D=D_{0} \exp (-E / R T) .
$$

As seen from Figure 3, the diffusion coefficient of both water and ethanol in the presence of a Pt surface obeys the Arrhenius equation; the calculated apparent 


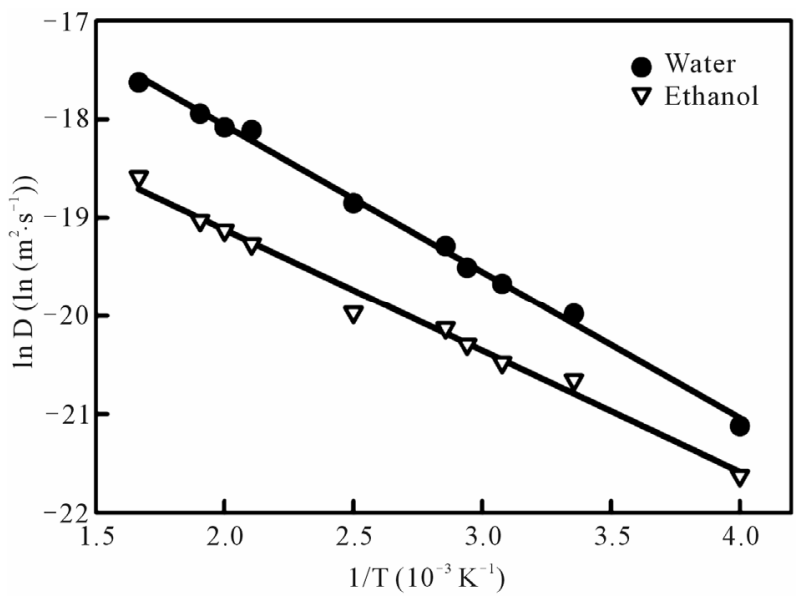

Figure 3. Relation between $\ln \mathrm{D}$ and reciprocal of temperature in Kelvin, solid line for water and the dashed line for ethanol, the inset figures represent the same relation at higher temperature range.

activation energies $(E)$ of diffusion are 2.47 and 2.98 $\mathrm{Kcal} / \mathrm{mole}$ for ethanol and water, respectively. These low E values explain the higher self-diffusion coefficients of both ethanol and water.

From our MD simulations, the diffusion coefficient is higher in the presence of a Pt surface than in its absence. Apparently, as experimental and theoretical results indicate, the diffusion coefficient is always higher in the presence of many metallic surfaces than in their absence. We observe a similar regularity in the presence of a $\mathrm{Pt}$ surface because it is a good catalyst of ethanol oxidation or dissociative adsorption. Such adsorption process makes the bulk of ethanol to adsorb on the surface, so it actively increases the mobility of the solution molecules toward the surface. In this aspect, a good correlation of our MD simulation results with experimental observations is clear.

It should be noted that all of the results presented above are constructed for a bulk solution, and they are not related specifically to the adsorbed molecules. It is well known that the 2D surface diffusion is different from that of a bulk solution.

\subsection{Intermolecular Structure}

The structure of liquids is ordinarily expressed in terms of radial distribution functions (RDF) $g(r)$. The most structured and interesting $g(r)$ functions for liquid ethanol correspond to the $\mathrm{O}-\mathrm{H}$ and $\mathrm{O}-\mathrm{O}$ bonding. Figures 4(a) and 4(b) show the OW-HW and Oe-He RDF graphs of $50: 50 \%$ ethanol-water mixtures. It is shown that Pt presence does not affect the RDF peak positions. However, the inclusion of a Pt surface notably affects the RDF amplitudes; the first peak at around $2 \AA$ is a strong evidence of hydrogen bonding in the bulk of the

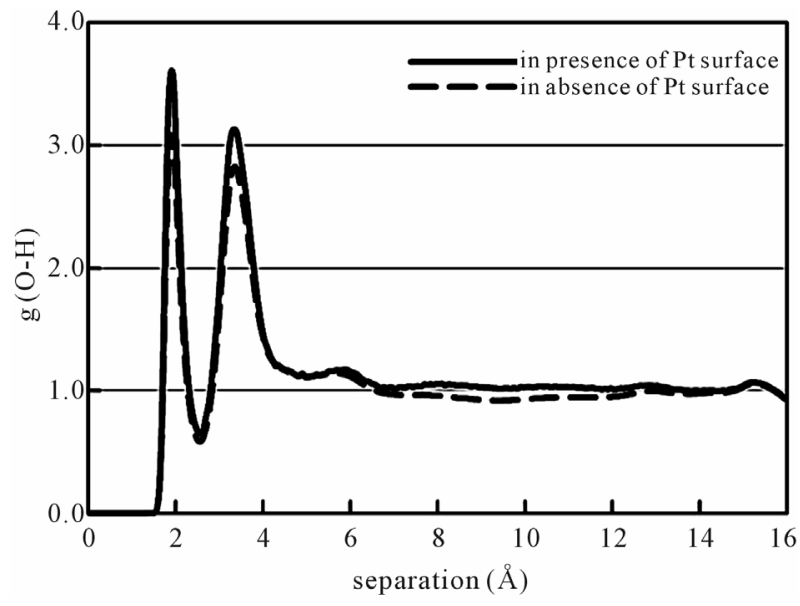

(a)

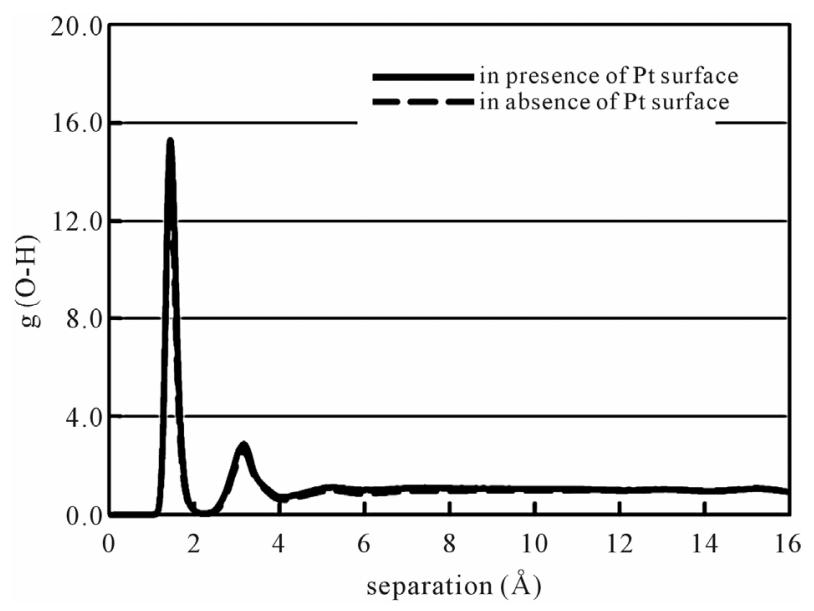

(b)

Figure 4. The radial distribution functions (RDF) of 50:50\% ethanol-water mixtures for OW-HW (a) and Oe-He (b). The solid line indicates the presence of a platinum surface; the dotted line corresponds to the absence of a platinum surface.

solution. Figures 5(a) and 5(b) represent RDF graphs for Oe-HW and OW-He atomic pairs. It is clear that a $\mathrm{Pt}$ surface enhances the value of $g(r)$, thereby making it easier for ethanol and water to approach each other and to establish a strong interaction between them. Such a strong ethanol-water interaction results in a more preferred ethanol-water structure formation. The O-O RDFs are shown in Figure 6, where O-O RDFs have maximum peaks at around $2.4-2.8 \AA$. In contrast to the $\mathrm{O}-\mathrm{H}$ RDFs, the second O-O peak located around 3.2 $3.5 \AA$ is not very obvious, indicating that there is a weak second correlation.

As we discussed before, the temperature of the system was raised by the annealing process by $25 \mathrm{~K}$ in each simulation, starting from $250 \mathrm{~K}$ to $600 \mathrm{~K}$. The RDF graphs representing the temperature effect on the waterwater, ethanol-ethanol and water-ethanol interactions 


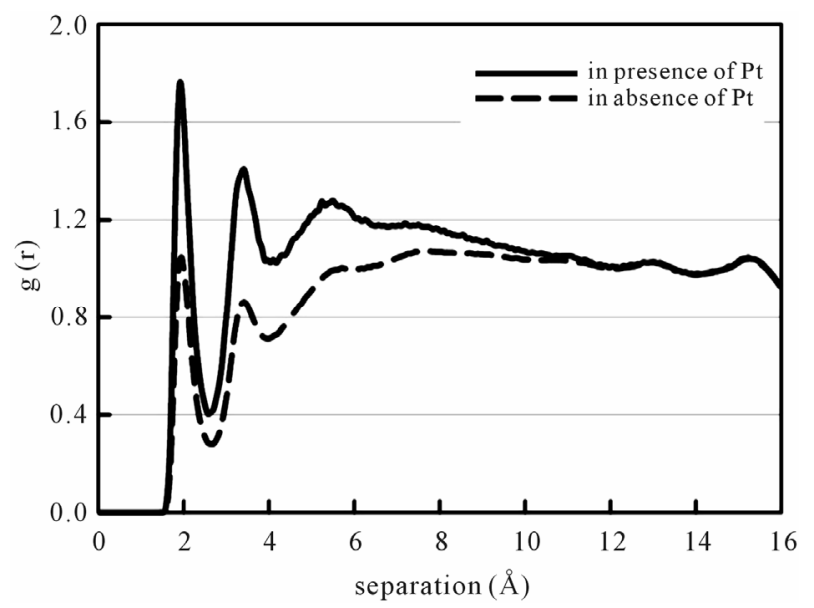

(a)

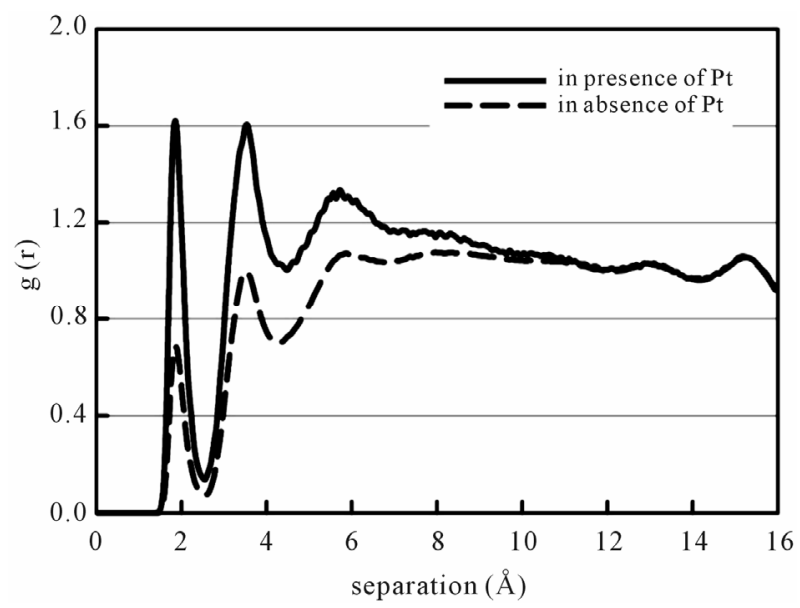

(b)

Figure 5. The radial distribution functions (RDF) of 50:50\% ethanol-water mixtures for Oe-HW (a) and OW-He (b). The solid line indicates the presence of a platinum surface; the dashed line corresponds to the absence of a platinum surface.

are shown in Figure 7. It is seen that the liquids become more structured when $T$ decreases. Also, the temperature changes do not affect significantly the peak positions, but only the height and depth of the peaks. The corresponding coordination numbers $N_{C}(r)$ for OW-He and Oe-HW, which are determined by the integration of the pair radial distribution functions, are plotted in the insets of (Figures 7(c) and 7(d), respectively). The O-H coordination number is associated with hydrogen bonding information in the mixtures [24] up to first valleys (around $2.5 \AA$ ). The $N_{C}(r)$ functions for OW-He and Oe-HW show a clear plateau at low temperatures, while at higher temperatures the $N_{C}(r)$ functions do not have a horizontal plateau in the region of $g(r)$ minima; their values decrease with increasing temperature. It is worth mentioning that the values of the $N_{C}(r)$ functions of both OW-He and Oe-HW are lower than unity, which can

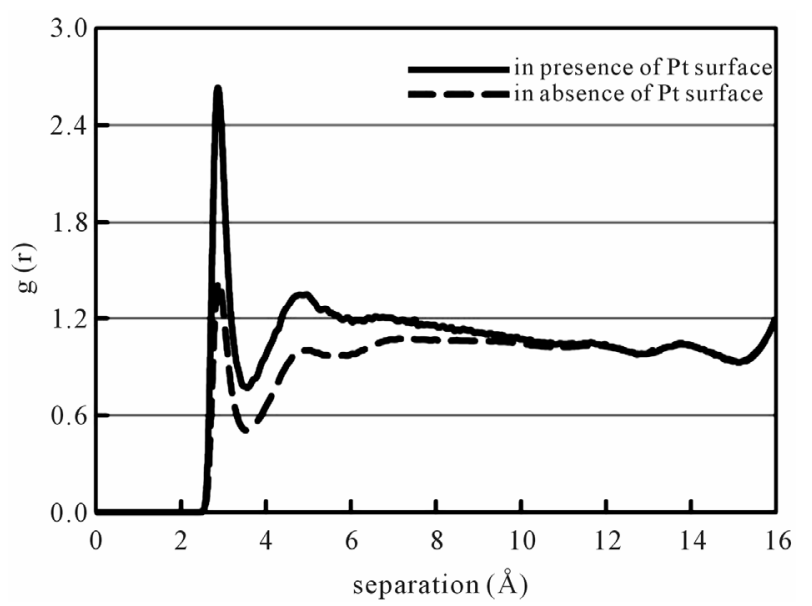

(a)

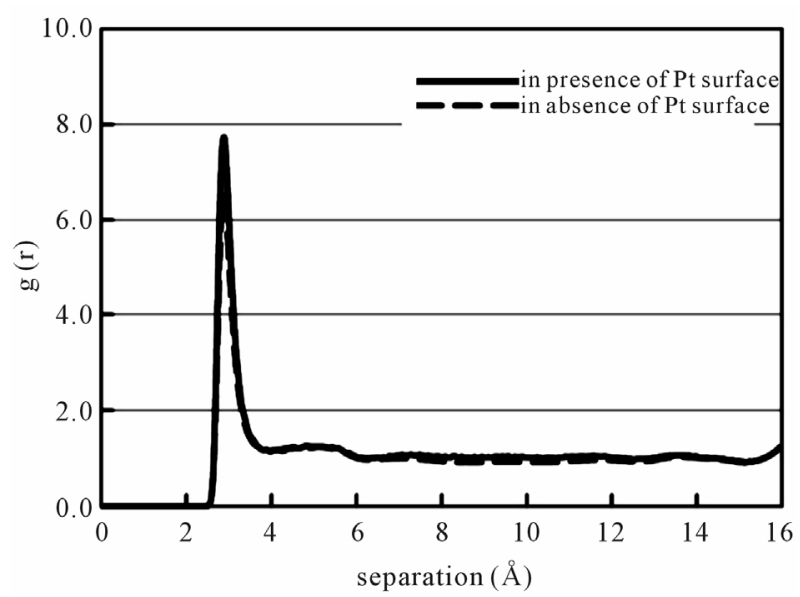

(b)

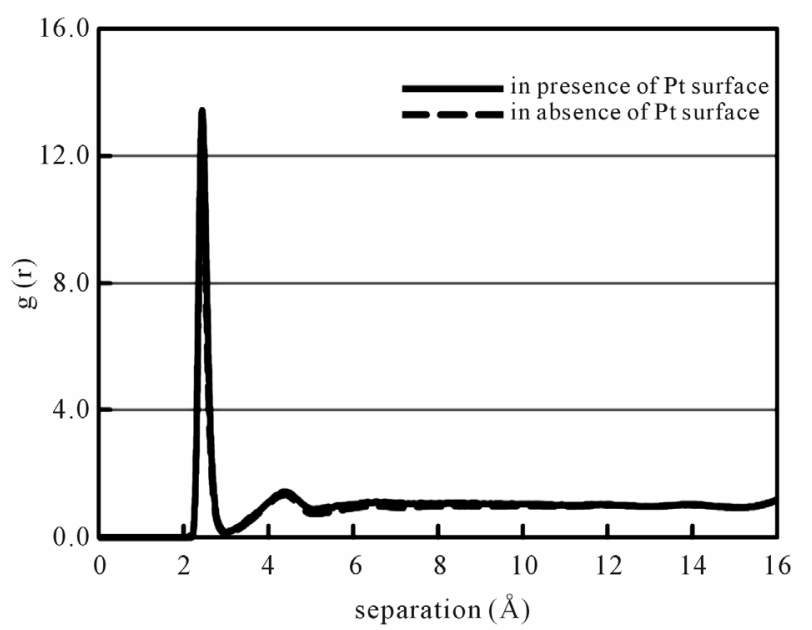

(c)

Figure 6. The radial distribution functions (RDF) of 50:50\% ethanol-water mixtures for OW-Oe (a), OW-OW (b) and Oe-Oe (c). The solid line indicates the presence of a platinum surface; the dashed line corresponds to the absence of a platinum surface. 


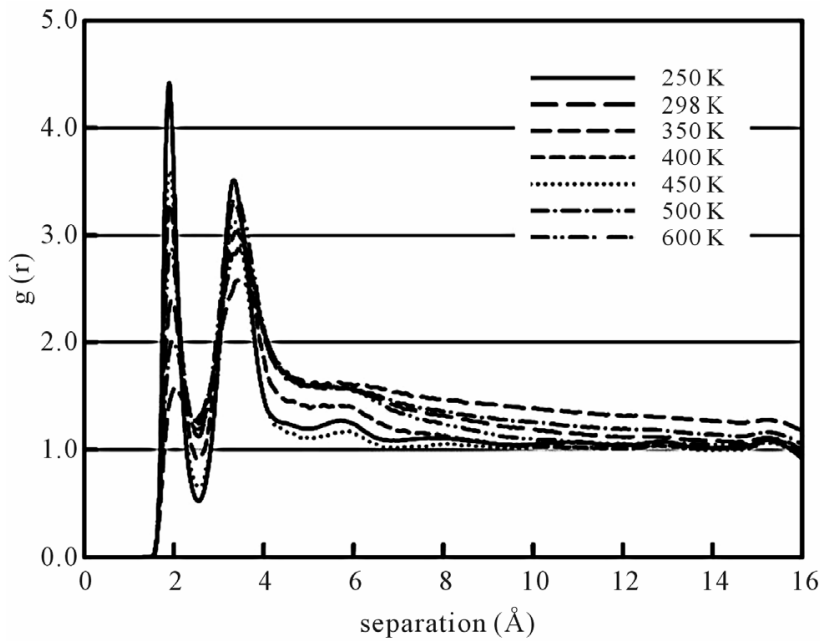

(a)

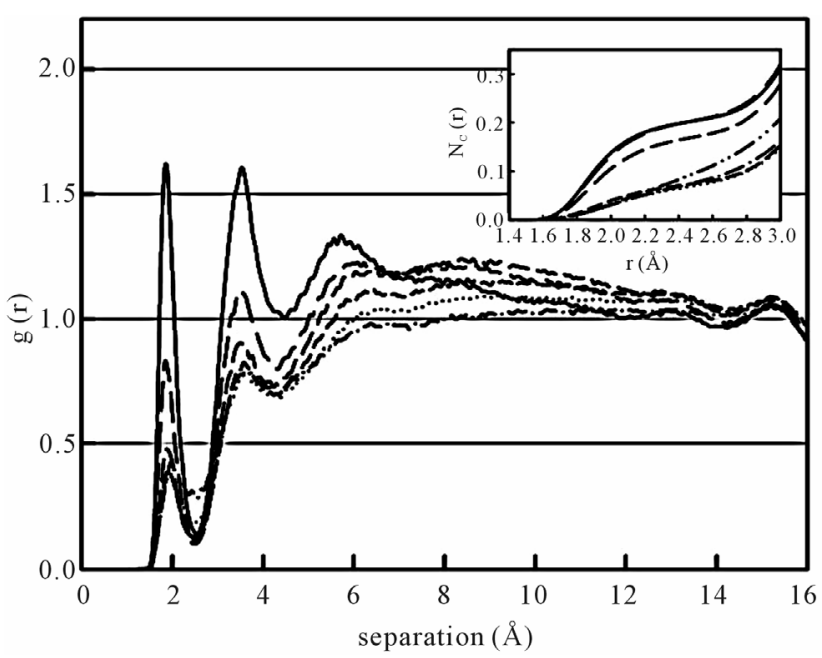

(c)

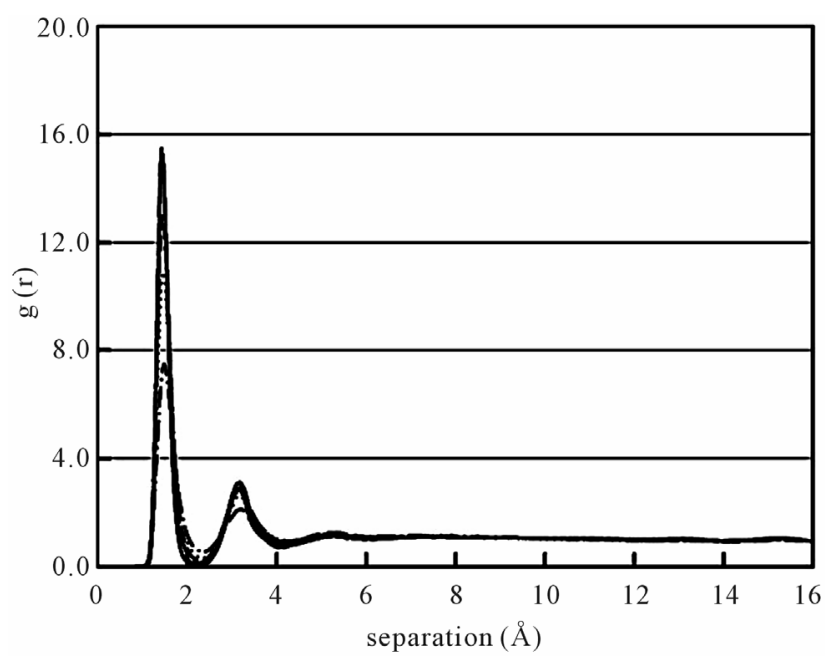

(b)

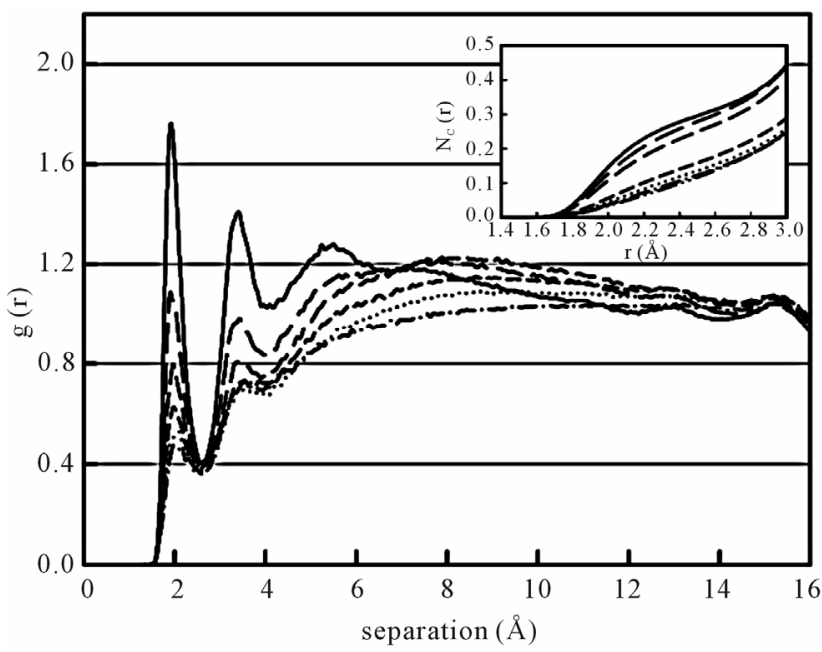

(d)

Figure 7. The effect of temperature on RDF graphs of (a) OW-HW; (b) Oe-He; (c) OW-He and (d) Oe-HW in presence of Pt surface and the corresponding $\mathrm{N}_{\mathrm{C}}(\mathrm{r})$ of $\mathrm{OW}-\mathrm{He}$ and Oe-HW, the temperature was raised by $25 \mathrm{~K}$ in each simulation starting from $250 \mathrm{~K}$ to $600 \mathrm{~K}$.

also be a proof of our last conclusion: the hydrogen networks between water and ethanol are disrupted.

\subsection{Interaction with Pt Surface}

During the molecular dynamics simulation, the interfacial structure of ethanol and water adsorption on $\mathrm{Pt}$ was analyzed by calculating the normalized ethanol and water density profiles as functions of the distance from a Pt surface. The first highly ordered adsorption layer is seen in Figure 8, where a simulation snapshot is shown together with a plot of the density of both ethanol and water molecules as a function of the perpendicular distance from the surface. From the density profile, there is a well-defined first adsorption layer from $\sim 2$ to $5 \AA$ and one more diffuse layer from $\sim 5$ to $8 \AA$. For distances beyond $\sim 10 \AA$, the relative density approaches unity as it would be expected in a bulk environment with no electrode influence. According to the present results, the interfacial region for ethanol adsorption on a Pt surface covers the range of $2-9 \AA$ with disorganization increasing towards the bulk liquid. On the other hand, it is observed that the relative density of water in the first adsorption layer is very low relative to that of ethanol, but for the second layer it is different. From this result, we can suggest that hydrogen networks between water and ethanol are disrupted as the solution mixture approaches the Pt surface. At a large distance from the surface, the relative density of the liquids-ethanol and water-approaches unity as it would be expected in a bulk environment with no electrode influence. The results 


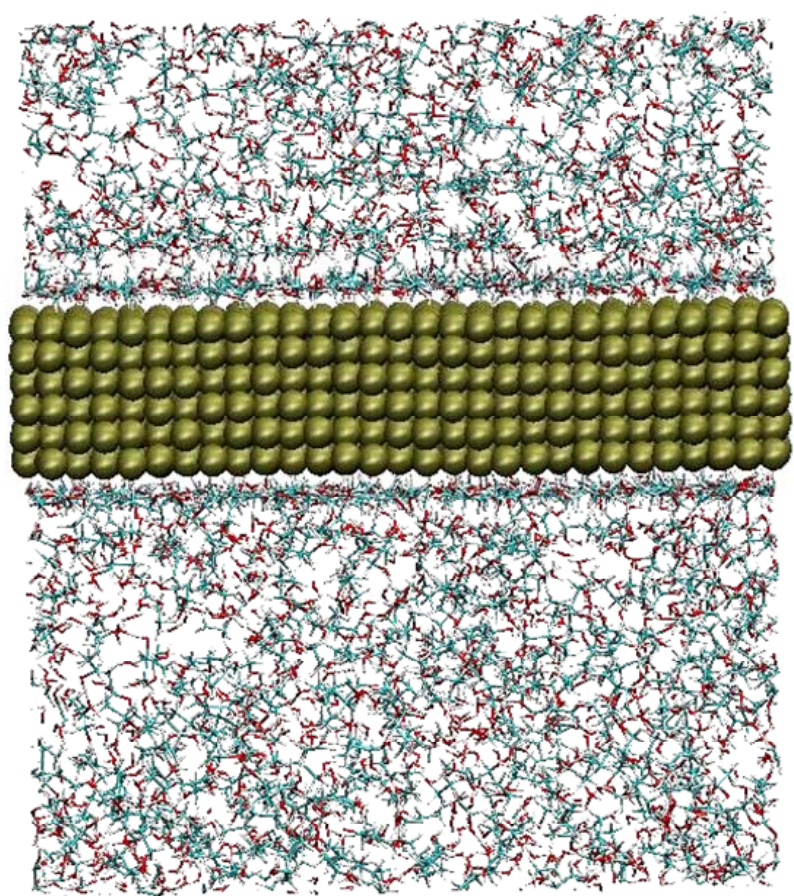

(a)

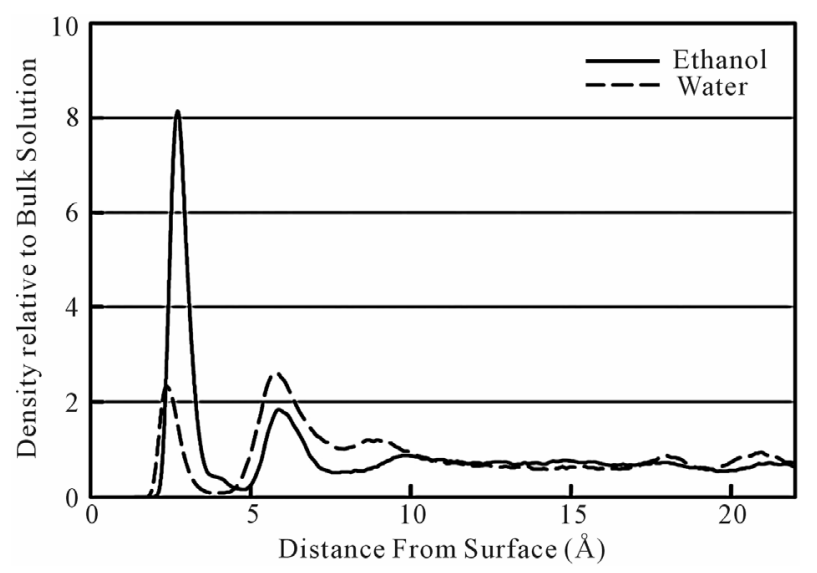

(b)

Figure 8. (a) Snapshot of the MD simulation of the waterethanol-Pt and (b) the density profile of ethanol-water system in presence of Pt-surface, the densities of both ethanol and water were normalized relative to the bulk density of solution which is about $0.8 \mathrm{~g} / \mathrm{cm}^{3}$.

also imply that ethanol molecules push the water molecules away from the surface, thereby forming a strongly adsorbed layer on the Pt surface. It is important to note that the density profile falls to zero between the adsorption peak and the secondary peak, which means there is little or no movement of ethanol molecules between the adsorption layer and the bulk solvent within the time scale of the simulation.

It is known that the diffusion coefficient of pure water or ethanol is larger than that of the 50:50 liquid. From the density profile illustrated above, the density of ethanol molecules on the first layer is higher than that of water molecules. But for the second layer it is different; the law is opposite. From this we can suggest that the hydrogen networks between water and ethanol are disrupted; then, near the surface, the liquid mixture is more like either water or ethanol. The diffusion coefficient will become larger. For the RDF, its amplitude in the 50:50 cases is larger. Thus, water-water or ethanolethanol molecule interaction will intensify, thereby governing the total system's dynamics.

The effect of temperature on the ethanol-Pt and water-Pt interaction is shown in Figure 9. As we discussed before, the liquids become more structured when $T$ decreases. Also, temperature changes affect only the peak amplitudes. By comparing $g(r)$ for Pt-Oe and that for Pt-OW, we can find that $g(r)$ for Pt-OW shows a marked decrease in the heights of peaks with increasing tempera-

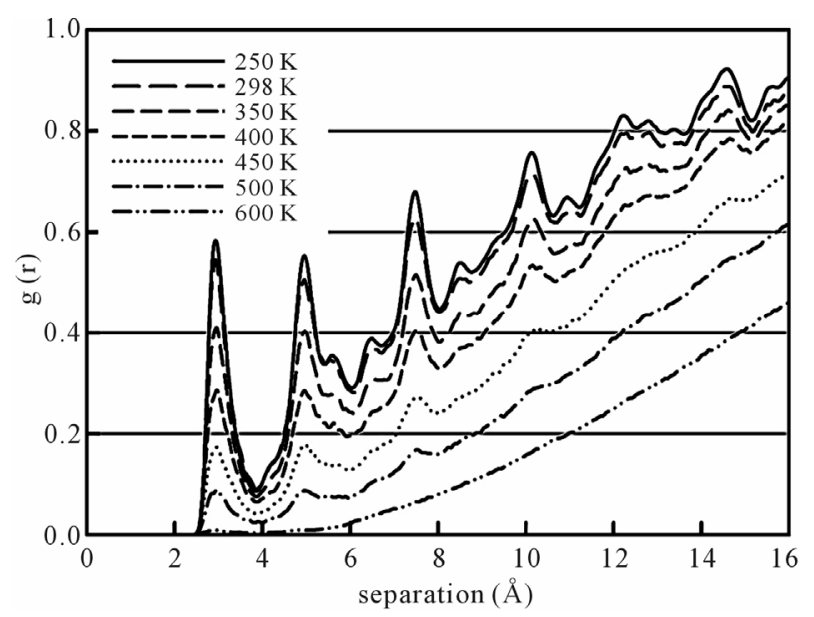

(a)

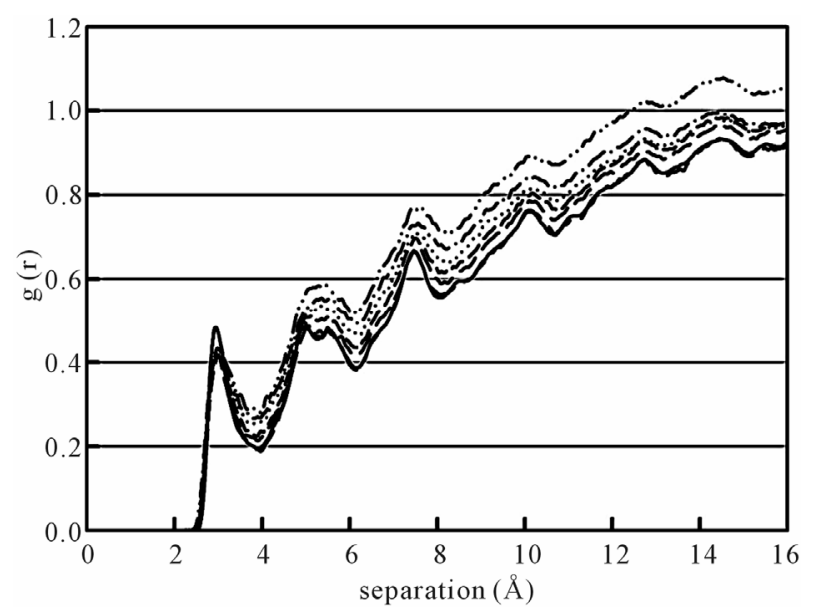

(b)

Figure 9. The effect of temperature on The RDF graphs of (a) Pt-Oe and (b) Pt-OW, the temperature was raised by $25 \mathrm{~K}$ in each simulation starting from $250 \mathrm{~K}$ to $600 \mathrm{~K}$. 
ture; finally, no peaks are observed in its RDF graph at $600 \mathrm{~K}$. In contrast to the RDF of Pt-OW, the heights of the peaks in the RDF graph of Pt-Oe do not behave so: only the height of the first peak slightly decreases, and the other peaks show slight increases in their heights with increasing temperature. All these results prove that at high temperatures most of water molecules get removed from the surface by ethanol molecules until only ethanol molecules are found at the Pt surface at $600 \mathrm{~K}$ (supplementary videos visualizing our system at $600 \mathrm{~K}$ are provided). The height of the first peak in the RDF graph of Pt-Oe slightly decreases with increasing temperature, which is due to the desorption process that can occur at higher temperatures.

\section{CONCLUSION REMARKS}

We simulated a water-ethanol mixture in the presence and absence of a Pt surface using DL_POLY version 2.19. The self-diffusion coefficients of both water and ethanol in the presence and absence of a Pt surface were calculated; an excellent agreement with the experimental results has been found within an error of 7.4\%. From our MD simulations the enhancement of the self-diffusion coefficients of both water and ethanol molecules related to the ethanol-water structure have been well-established in the presence of a Pt surface. As experimental and theoretical results indicate, the diffusion coefficient is always higher in the presence of many metallic surfaces than in their absence. The temperature of the system was varied using annealing process and its effects on selfdiffusion coefficient and radial distribution functions (RDF) graphs were illustrated. The RDF graphs in addition to the density profile have been built, and RDF correlations with the self-diffusion coefficients of both ethanol and water molecules are illustrated.

\section{ACKNOWLEDGEMENTS}

This work has been performed in the framework of joint collaborative agreement Arab Republic of Egypt (ARE) - Joint Institute for Nuclear Research (JINR) (project \#302 "Molecular Dynamics Research of Radiobiological Problems"). This work was supported in part by the Grant in Aid for the Global Center of Excellence Program of the Center for Education and Research of Symbiotic, Safe and Secure System Design from Japan's Ministry of Education, Culture, Sport, and Technology.

Supporting Information Available: A visualization video of our system, ethanol-water in presence of a Pt surface, at $600 \mathrm{~K}$, this video was created using VMD program. This material is available free of charge via the internet at http://pubs.acs.org

\section{REFERENCES}

[1] Carlos, A.C. and Óscar, J.S. (2007) Fuel ethanol produc- tion: process design trends and integration opportunities. Bioresource Technology, 98, 2415-2457.

doi:10.1016/j.biortech.2007.01.002

[2] Carlos, A.C. and Óscar, J.S. (2008) Trends in biotechnological production of fuel ethanol from different feedstocks. Bioresource Technology, 99, 5270-5295. doi:10.1016/j.biortech.2007.11.013

[3] Shen, S.Y., Zhao, T.S. and Xu, J.B. (2010) Carbon supported PtRh catalysts for ethanol oxidation in alkaline direct ethanol fuel cell. International Journal of Hydrogen Energy, 35, 12911-12917. doi:10.1016/j.ijhydene.2010.08.107

[4] Vasudevan, V.N. and Leland, M.V. (2007) High permeability membranes for the dehydration of low water content ethanol by pervaporation. Journal of Membrane Science, 306, 209-215.

doi:10.1016/j.memsci.2007.08.050

[5] Song, S.Q. and Tsiakaras, P. (2006) Recent progress in direct ethanol proton exchange membrane fuel cells (DEPEMFCs). Applied Catalysis B, 63, 187-193. doi:10.1016/j.apcatb.2005.09.018

[6] Song, S.Q., Zhou, W.J., Zhou, Z.H., Jiang, L.H., Sun, G.Q., Xin, Q., Leontidis, V., Kontou, S. and Tsiakaras, P. (2005) Direct ethanol PEM fuel cells: The case of platinum based anodes. International Journal of Hydrogen Energy, 30, 995-1001.

doi:10.1016/j.ijhydene.2004.11.006

[7] Yao-Chun, W., Chaun, C. and Shin-Pon, J. (2008) Adsorption mechanism and dynamic behavior of water and ethanol molecules inside Au nanotubes. Chinese Journal of Catalysis, 29, 1099-1106. doi:10.1016/S1872-2067(09)60008-5

[8] Kousksou, T., Jamil, A., Zeraouli, Y. and Dumas, J.P. (2007) Equilibrium liquidus temperatures of binary mixtures from differential scanning calorimetry. Chemical Engineering Science, 62, 6516-6523. doi:10.1016/j.ces.2007.07.008

[9] Zhang, C., and Yang, X. (2005) Molecular dynamics simulation of ethanol/water mixtures for structure and diffusion properties. Fluid Phase Equilibria, 231, 1-10. doi:10.1016/j.fluid.2005.03.018

[10] Kusalik, P.G., Lyubarts, A.P., Bergman, D.L. and Laaksonen, A. (2000) Computer simulation study of tertbutyl alcohol. Structure in aqueous solution. Journal of Physical Chemistry B, 104, 9533-9539. doi:10.1021/jp001887o

[11] Soper, A.K. and Finney, J.L. (1993) Hydration of methanol in aqueous solution. Physical Review Letters, 71, 4346-4350. doi:10.1103/PhysRevLett.71.4346

[12] Sachtler, W.M.H. and Ichikawa, M. (1986) Catalytic site requirements for elementary steps in syngas conversion to oxygenates over promoted rhodium. Journal of Physicla Chemistry, 90, 4758-4764. doi:10.1021/j100411a009

[13] Ahmed, G., Atta, N.F., Darwesh, S.A. and Ali S.M. (2008) Electrodeposited metals at conducting polymer electrodes. II: Study of the oxidation of methanol at poly (3-methylthiophene) modified with Pt-Pd Co-catalyst. Topics in Catalysis, 47, 73-83.

[14] Kholmurodov, Kh., Puzynin, I., Smith, W., Yasuoka, K. and Ebisuzaki, T. (2001) MD simulation of cluster-surface impacts for metallic phases: soft landing, droplet 
spreading and implantation. Computer Physics Communications, 141, 1-16.

doi:10.1016/S0010-4655(01)00292-2

[15] David, C.Y. (2001) Computational Chemistry: A practical guide for applying techniques to real-world problems. Chapter 7John Wiley \& Sons, Inc., New York.

[16] Cooke, D.J., Gray, R.J., Sand, K.K., Stipp, S.L.S. and Elliott, J.A. (2010) Interaction of ethanol and water with the $\{1014\}$ surface of calcite. Langmuir, 26, 1452014529. doi:10.1021/la100670k

[17] Smith, W. and Forester, T.R. (1996) A general-purpose parallel molecular dynamics simulation package. Journal of Molecular Graphics, 14, 136-141. doi:10.1016/S0263-7855(96)00043-4

[18] Smith, W., Forester, T.R. and Todorov, I.T. (2008) The DL POLY 2 User Manual, Version 2.19. STFC Daresbury Laboratory, Daresbury, Warrington WA4 4AD Cheshire, UK.

[19] Sutton, A.P. and Chen, J. (1990) Long-range FinnisSinclair potential. Philosophical Magazine Letters, 61, 139-146. doi: $10.1080 / 09500839008206493$
[20] WWW-MINCRYST, Crystallographic and crystallochemical database for minerals and their Structural Analogues. http://database.iem.ac.ru/mincryst/

[21] Price, W.S., Ide, W.S. and Arata, Y. (2003) Solution dynamics in aqueous monohydric alcohol systems. Journal of Physical Chemistry A, 107, 4784-4789. doi:10.1021/jp027257z

[22] Wensink, E.J.W., Hoffmann, A.C., van Maaren, P.J. and van der Spoel, D. (2003) Dynamic properties of water/alcohol mixtures studied by computer simulation. Journal of Chemical Physics, 119, 7308-7317. doi:10.1063/1.1607918

[23] Li, W., Chen, C. and Yang, J. (2008) Molecular dynamics simulation of self-diffusion coefficient and its relation with temperature using simple Lennard-Jones potential. Heat Transfer-Asian Research, 37, 86-93.

[24] Dixit, S., Crain, J., Poon, W.C.K., Finney, J.L. and Soper, A.K. (2002) Molecular segregation observed in a concentrated alcohol-water solution. Nature, 416, 829-832. doi: $10.1038 / 416829 \mathrm{a}$ 